\title{
On the $l$-divisibility of the relative class number of certain cyclic number fields
}

\author{
by
}

KurT Girstmair (Innsbruck)

Introduction. Let $q$ be a natural number and $p$ a prime with $2 q \mid p-1$. Let $\xi_{p}=e^{2 \pi i / p}$ and $\mathbb{Q}_{p}=\mathbb{Q}\left(\xi_{p}\right)$, i.e., the $p$ th cyclotomic field. Moreover, consider $\mathbb{F}_{p}=\mathbb{Z} / p \mathbb{Z}=\{\overline{0}, \overline{1}, \ldots, \overline{p-1}\}$ and the multiplicative group $G_{p}=$ $\mathbb{F}_{p}^{\times}$of this field. There is a canonical group isomorphism

$$
G_{p} \rightarrow \operatorname{Gal}\left(\mathbb{Q}_{p} / \mathbb{Q}\right): k \mapsto \sigma_{k},
$$

$\sigma_{k}$ being defined by $\sigma_{k}\left(\xi_{p}\right)=\xi_{p}^{k}$. The field $\mathbb{Q}_{p}$ contains a uniquely determined subfield $K_{2 q}=K_{2 q, p}$ of degree $\left[K_{2 q}: \mathbb{Q}\right]=2 q$, viz., the fixed field of the group $\left\{\sigma_{k} ; \bar{k} \in G_{p}^{2 q}\right\}$. Here $G_{p}^{m}$ means $\left\{\bar{k}^{m} ; \bar{k} \in G_{p}\right\}, m \in \mathbb{N}$. $K_{2 q}$ is imaginary if and only if $-1 \notin G_{p}^{2 q}$, i.e., $p \equiv 2 q+1 \bmod 4 q$. We shall assume this throughout the present paper.

In the sequel let $g=g_{p} \in G_{p}$ be chosen such that

$$
G_{p} / G_{p}^{2 q}=\left\{\overline{1}, \bar{g}, \ldots, \bar{g}^{q-1},-\overline{1},-\bar{g}, \ldots,-\bar{g}^{q-1}\right\} .
$$

This holds, e.g., if one of the following assumptions is fulfilled:

Assumption A. $\langle\bar{g}\rangle=G_{p} / G_{p}^{2 q}$.

Assumption B. $q$ is odd and $\langle\bar{g}\rangle=G_{p}^{2} / G_{p}^{2 q}$.

The reader may verify (1) in both cases. Now let $t \in G_{p} / G_{p}^{2 q}$. Thus $t$ is a set of elements of $G_{p}$, and we define the excess $\Phi_{t}$ of this set by

$$
\Phi_{t}=|\{k ; 1 \leq k<p / 2, \bar{k} \in t\}|-|\{k ; p / 2<k \leq p-1, \bar{k} \in t\}| .
$$

If $g=g_{p}$ is as above and $j \in \mathbb{Z}$, we put, in particular,

$$
\Phi_{j}=\Phi_{j}(g)=\Phi_{\bar{g}^{j}}=\left|\left\{k<p / 2 ; \bar{k} \in \bar{g}^{j}\right\}\right|-\left|\left\{k>p / 2 ; \bar{k} \in \bar{g}^{j}\right\}\right| .
$$

Then

$$
\Phi=\Phi(g)=\left(\Phi_{0}, \ldots, \Phi_{q-1}\right) \in \mathbb{Z}^{q}
$$

is called the excess vector belonging to $g=g_{p}$. Because of (1) and the relation $\Phi_{-t}=-\Phi_{t}$, the vector $\Phi$ describes all excesses $\Phi_{t}, t \in G_{p} / G_{p}^{2 q}$. 
In the subsequent Section 1 we express the relative class number $h_{2 q}^{-}=$ $h_{2 q}^{-}(p)$ of the field $K_{2 q}$ in terms of the excesses $\Phi_{j}, j=0, \ldots, q-1$ (formulas $(2),(4 \mathrm{~A}),(4 \mathrm{~B}))$. Thereby we generalize formulas given in [4].

In Section 2 we investigate the divisibility of $h_{2 q}^{-}$by an odd prime number $l$. The assertion $l \mid h_{2 q}^{-}$can be rephrased in systems of linear congruences $\bmod l$ for the excesses $\Phi_{0}, \ldots, \Phi_{q-1}$ (Theorems 1,2). More precisely, the following holds: Suppose that for all primes $p \equiv 2 q+1 \bmod 4 q$ the element $g=g_{p}$ is chosen such that Assumption A is satisfied. Then there exists, for almost all primes $l$, a linear manifold $M_{l} \subseteq \mathbb{F}_{l}^{q}$, i.e., a union of finitely many linear subspaces of $\mathbb{F}_{l}^{q}$, with the following property: $l$ divides $h_{2 q}^{-}(p)$ if and only if $\bar{\Phi}(g)=\left(\bar{\Phi}_{0}, \ldots, \bar{\Phi}_{q-1}\right)\left(\in \mathbb{F}_{l}^{q}\right)$ is in $M_{l}$ (Theorem 3). The corresponding result is also valid under Assumption B.

In Section 3 we consider special cases in which the congruences describing $M_{l}$ can be rendered in a completely explicit shape. Some of these results have been found previously, but from a less general viewpoint (cf. [4]).

Section 4 is based on the following plausible (yet unproved) hypothesis: In the situation of Theorem 3 we suppose that the excess vectors $\bar{\Phi}(g)$ are equally distributed in the space $\mathbb{F}_{l}^{q}$ when $p$ runs through all primes $\equiv 2 q+1 \bmod 4 q$. Then

$$
m_{l}=\left|M_{l}\right| / l^{q}
$$

is the probability that an arbitrary vector $\bar{\Phi}(g)$ is in $M_{l}$. By Theorem 3, this is the probability that $l$ divides $h_{2 q}^{-}(p)$. For $1 \leq q \leq 6$ and $3 \leq l<100$ we compare $m_{l}$ with the number

$$
n_{l}=\frac{\left|\left\{p<500000 ; p \equiv 2 q+1 \bmod 4 q, l \mid h_{2 q}^{-}(p)\right\}\right|}{|\{p<500000 ; p \equiv 2 q+1 \bmod 4 q\}|} .
$$

The result is given in Table 1 , and it shows a high degree of conformity between $m_{l}$ and $n_{l}$ in most cases.

At the end of this paper we give a table of the numbers $h_{12}^{-}(p), p<10000$. The corresponding tables for $h_{2 q}^{-}(p), 1 \leq q \leq 5$, can be found in [2], [6], and [3].

1. Formulas for $h_{2 q}^{-}$. Let the above notations hold. By $X_{2 q}$ we denote the character group of $G_{p} / G_{p}^{2 q}$; as usual, we consider $X_{2 q}$ as a subgroup of the character group of $G_{p}$, viz.,

$$
X_{2 q}=\left\{\chi ; \operatorname{Ker} \chi \supseteq G_{p}\right\} .
$$

Let $X_{2 q}^{-}$be the set of odd characters in $X_{2 q}$. Then $\left|X_{2 q}^{-}\right|=q$. Suppose that $g$ satisfies (1). For a vector $a=\left(a_{0}, \ldots, a_{q-1}\right) \in \mathbb{C}^{q}$ we define the Fourier transform

$$
F a=\left((F a)_{\chi} ; \chi \in X_{2 q}^{-}\right) \in \mathbb{C}^{X_{2 q}^{-}}
$$


by its components

$$
(F a)_{\chi}=\sum_{j=0}^{q-1} \chi\left(g^{j}\right) a_{j} .
$$

For the special vector $a=\Phi=\Phi(g)$ the transform $F \Phi$ is independent of the choice of $g$. Indeed,

$$
(F \Phi)_{\chi}=\sum(\chi(\bar{k}) ; 1 \leq k<p / 2)
$$

(cf. [4], Lemma 1). As in [4] one obtains

$$
\prod\left((F \Phi)_{\chi} ; \chi \in X_{2 q}^{-}\right)=\prod\left((\chi(\overline{2})-2) B_{\chi} ; \chi \in X_{2 q}^{-}\right)
$$

$B_{\chi}$ being the first Bernoulli number attached to $\chi$. In order to evaluate the product on the right side, one needs the order $f_{q}\left(f_{2 q}\right.$, resp.) of the element $\overline{\overline{2}}$ in the group $G_{p} / G_{p}^{q}\left(G_{p} / G_{p}^{2 q}\right.$, resp.). For each prime $p \equiv 2 q+1 \bmod 4 q$, $p>2 q+1$, the fundamental formula

$$
\prod\left((F \Phi)_{\chi} ; \chi \in X_{2 q}^{-}\right)=2^{q-1} C_{2 q} h_{2 q}^{-}
$$

holds, with

$$
C_{2 q}=C_{2 q}(p)=\left(2^{f_{q}}+(-1)^{f_{2 q} / f_{q}}\right)^{q / f_{q}}
$$

(cf. [4], Theorem 1 and formula (9)).

As to the actual computation of the relative class number, it is useful to write the left side of (2) as a determinant in terms of the excesses $\Phi_{j}$. First suppose that Assumption A of the Introduction holds. Let the character $\psi \in X_{2 q}^{-}$be arbitrarily chosen. Then

$$
\operatorname{det}\left(\psi\left(g^{j-k}\right) \Phi_{j-k} ; j, k=0, \ldots, q-1\right)=2^{q-1} C_{2 q} h_{2 q}^{-} .
$$

In the case of Assumption B one has the simpler formula

$$
\operatorname{det}\left(\Phi_{j-k} ; j, k=0, \ldots, q-1\right)=2^{q-1} C_{2 q} h_{2 q}^{-} .
$$

Indeed, the determinants in question are group determinants for the group $G_{p} / G_{p}^{q}$. Their evaluation is well-known (cf. [5], p. 23) and, together with (2), yields $(4 \mathrm{~A})$ and $(4 \mathrm{~B})$. These formulas have been used for the numerical computations displayed in Section 4.

2. Divisibility of $h_{2 q}^{-}$and congruences for the excesses. In what follows let $q$ be a natural number, $p$ a prime, $p \equiv 2 q+1 \bmod 4 q, p>2 q+1$. In addition, let $l$ be an odd prime not dividing $q$. The values of each character $\chi \in X_{2 q}^{-}$are in the field $\mathbb{Q}_{2 q}=\mathbb{Q}\left(\xi_{2 q}\right), \xi_{2 q}=e^{\pi i / q}$. We consider the automorphism $\tau_{l} \in \operatorname{Gal}\left(\mathbb{Q}_{2 q} / \mathbb{Q}\right)$ defined by

$$
\tau_{l}\left(\xi_{2 q}\right)=\xi_{2 q}^{l} \text {. }
$$


For each $\chi \in X_{2 q}^{-}$the map $\tau_{l} \circ \chi: G_{p} \rightarrow \mathbb{C}^{\times}: \bar{k} \mapsto \tau_{l}(\chi(\bar{k}))$ is in $X_{2 q}^{-}$again. Hence the group $\left\langle\tau_{l}\right\rangle$ acts on the set $X_{2 q}^{-}$. The orbits under this action will play an important role.

The group $\left\langle\tau_{l}\right\rangle$ is the decomposition group of $l$ in $\mathbb{Q}_{2 q}$, and $L=L_{l}\left(\subseteq \mathbb{Q}_{2 q}\right)$ denotes its fixed field. Let $\mathfrak{L}$ be a prime ideal of $\mathbb{Q}_{2 q}$ with $\mathfrak{L} \mid l$ and put $\mathfrak{l}=\mathfrak{L} \cap L$. Since $l$ splits completely in $L, \mathfrak{l}$ is a prime ideal of degree 1 over $\mathbb{Q}$. We denote by $\mathcal{O}_{2 q}\left(\mathcal{O}_{L}\right.$, resp.) the ring of integers of $\mathbb{Q}_{2 q}$ (of $L$, resp.). The canonical maps

$$
\begin{aligned}
\mathbb{F}_{l} & \rightarrow \mathcal{O}_{L} / \mathfrak{l}, & \mathcal{O}_{L} / \mathfrak{l} & \rightarrow \mathcal{O}_{2 q} / \mathfrak{L} \\
\bar{k} & \mapsto \bar{k} & \bar{x} & \mapsto \bar{x}
\end{aligned}
$$

allow us to identify $\mathcal{O}_{L} / \mathfrak{l}$ with $\mathbb{F}_{l}$ and to consider $\mathbb{F}_{l}$ as a subset of $\mathcal{O}_{2 q} / \mathfrak{L}$.

THEOREM 1. In the above situation, the following assertions are equivalent:

(i) $C_{2 q} h_{2 q}^{-} \equiv 0 \bmod l$.

(ii) There is a prime divisor $\mathfrak{L}$ of $l$ in $\mathbb{Q}_{2 q}$ and a character $\chi \in X_{2 q}^{-}$such that $(F \Phi)_{\chi} \equiv 0 \bmod \mathfrak{L}$.

(iii) There is a prime divisor $\mathfrak{L}$ of $l$ in $\mathbb{Q}_{2 q}$ and an orbit $Y=\left\langle\tau_{l}\right\rangle \circ \chi_{1}$ $\left(\subseteq X_{2 q}^{-}\right)$such that, for all $\chi \in Y,(F \Phi)_{\chi} \equiv 0 \bmod \mathfrak{L}$.

Proof. The equivalence of (i) and (ii) is an immediate consequence of formula (2). Because of $\tau_{l}(\mathfrak{L})=\mathfrak{L}$, assertion (iii) is equivalent to (ii).

The congruence $(F \Phi)_{\chi} \equiv 0 \bmod \mathfrak{L}$ can be considered as an equation over the field $\mathcal{O}_{2 q} / \mathfrak{L}$, of course. Then (iii) says that $\bar{\Phi}=\left(\bar{\Phi}_{0}, \ldots, \bar{\Phi}_{q-1}\right) \in \mathbb{F}_{l}^{q}$ is a solution of the system of linear equations

$$
\sum_{j=0}^{q-1} \overline{\chi\left(g^{j}\right)} \bar{\Phi}_{j}=\overline{0}, \quad \chi \in Y,
$$

with coefficients $\overline{\chi\left(g^{j}\right)}$ in $\mathcal{O}_{2 q} / \mathfrak{L}$. In the next theorem we transform (5) into an equivalent system with coefficients in $\mathbb{F}_{l}$ and determine its rank. For this purpose we need the trace map

$$
T_{l}: \mathbb{Q}_{2 q} \rightarrow L_{l}: x \mapsto \sum\left(\tau(x) ; \tau \in\left\langle\tau_{l}\right\rangle\right) .
$$

By $\varphi$ we denote Euler's function, as usual.

THEOREM 2. In the situation above suppose that $l \nmid \varphi(q)$. Let $\chi_{1} \in X_{2 q}^{-}$ and $Y=\left\langle\tau_{l}\right\rangle \circ \chi_{1}$. The vector $\bar{\Phi}=\left(\bar{\Phi}_{0}, \ldots, \bar{\Phi}_{q-1}\right) \in \mathbb{F}_{l}^{q}$ is a solution of (5) if and only if it is a solution of the system

$$
\sum_{j=0}^{q-1} \overline{T_{l}\left(\chi_{1}(g)^{j-k}\right)} \bar{\Phi}_{j}=\overline{0}, \quad k=0, \ldots,|Y|-1,
$$


with coefficients in $\mathcal{O}_{L} / \mathfrak{l}=\mathbb{F}_{l}$. The dimension of the space $V_{Y, g}$ of solutions of (6) is $q-|Y|$.

Proof. Suppose that $r=|Y|$ and $Y=\left\{\chi_{1}, \ldots, \chi_{r}\right\}$. By means of the Fourier transform of Section 1 we define the linear map

$$
\lambda:\left(\mathcal{O}_{2 q} / \mathfrak{L}\right)^{q} \rightarrow\left(\mathcal{O}_{2 q} / \mathfrak{L}\right)^{r}: \bar{a} \mapsto\left(\overline{(F a)_{\chi_{1}}}, \ldots, \overline{(F a)_{\chi_{r}}}\right) .
$$

The matrix of $\lambda$ (with respect to the standard bases) is

$$
A=\left(\overline{\chi_{i}\left(g^{j}\right)} ; i=1, \ldots, r, j=0, \ldots, q-1\right) .
$$

Because of (1), $G_{p} / G_{p}^{2 q}=\langle-\overline{\overline{1}}, \bar{g}\rangle$, which implies that the values $\chi_{i}(g), i=$ $1, \ldots, r$, are all different. Moreover, $l$ does not divide $2 q$, hence the $2 q$ th roots of unity $\overline{\chi_{i}(g)}$ are all different, too. This means that the minor $\left(\overline{\chi_{i}\left(g^{j}\right)} ; i=\right.$ $1, \ldots, r, j=0, \ldots, r-1$ ) of $A$ is a regular matrix (of Vandermonde type). Therefore the rank of $A$ is $r$ and $\lambda$ is surjective. Let $c$ be the natural number

$$
c=\operatorname{ord}\left(\tau_{l}\right) / r,
$$

with $\operatorname{ord}\left(\tau_{l}\right)=\left|\left\langle\tau_{l}\right\rangle\right|$. Since $\varphi(2 q)=\left[\mathbb{Q}_{2 q}: \mathbb{Q}\right] \not \equiv 0 \bmod l, \bar{c} \in \mathbb{F}_{l}$ is different from $\overline{0}$. We define another linear map

$$
\mu:\left(\mathcal{O}_{2 q} / \mathfrak{L}\right)^{r} \rightarrow\left(\mathcal{O}_{2 q} / \mathfrak{L}\right)^{r}
$$

by putting

$$
\mu\left(\bar{b}_{1}, \ldots, \bar{b}_{r}\right)=\left(\bar{c} \sum_{i=1}^{r} \overline{\chi_{i}\left(g^{-j}\right)} \bar{b}_{i} ; j=0, \ldots, r-1\right) .
$$

The matrix of $\mu$ (with respect to the standard bases) is

$$
B=\left(\bar{c} \overline{\chi_{i}\left(g^{-j}\right)} ; j=0, \ldots, r-1, i=1, \ldots, r\right) .
$$

By the above, $B$ is regular and $\mu$ bijective. Thus $\mu \circ \lambda$ is surjective. The $k$ th component of $\mu \circ \lambda(\bar{a})$ is

$$
(\mu \circ \lambda(\bar{a}))_{k}=\sum_{j=0}^{q-1} \bar{c} \sum_{i=1}^{r} \overline{\chi_{i}\left(g^{j-k}\right)} \bar{a}_{j}=\sum_{j=0}^{q-1} \overline{T_{l}\left(\chi_{1}\left(g^{j-k}\right)\right)} \bar{a}_{j},
$$

$k=0, \ldots, r-1$. Now $\bar{\Phi}$ is in the space $V_{Y, g}$ of solutions if and only if $\lambda(\bar{\Phi})=0$. Since $\mu$ is bijective, this is equivalent to $\mu \circ \lambda(\bar{\Phi})=0$. By (7), this means that $\bar{\Phi}$ is a solution of (6).

Finally, observe that the matrix of $\mu \circ \lambda$ is

$$
B A=\left(\overline{T_{l}\left(\chi_{1}\left(g^{j-k}\right)\right)} ; k=0, \ldots, r-1, j=0, \ldots, q-1\right) .
$$

Its coefficients are in $\mathcal{O}_{L} / \mathfrak{l}=\mathbb{F}_{l}$, and the fact that $\mu \circ \lambda$ is surjective shows that its rank is $r$. Thus $V_{Y, g}$ has dimension $q-r=q-|Y|$. 
R e m a r k. Theorem 2 can be rephrased without the assumption $l \nmid \varphi(q)$. But then the trace $T_{l}$ must be replaced by a trace $T_{l, Y}: L_{l, Y} \rightarrow L_{l}$, where $L_{l, Y}$ is a subfield of $\mathbb{Q}_{2 q}$ depending on $l$ and $Y$.

Let $\mathcal{Y}$ be the set of all orbits $Y$ of the group $\left\langle\tau_{l}\right\rangle$ on $X_{2 q}^{-}$. We define the linear manifold

in $\mathbb{F}_{l}^{q}$ and show

$$
M_{l, g}=\bigcup\left(V_{Y, g} ; Y \in \mathcal{Y}\right)
$$

Lemma 1. Let $p$ run through all primes $\equiv 2 q+1 \bmod 4 q, p>2 q+1$, and suppose that the elements $g=g_{p}$ are chosen such that Assumption A of the Introduction holds. Then $M_{l, g}$ is independent of the choice of $g$ and $p$.

Proof. Let

$$
E_{2 q}^{-}=\left\{\eta \in \mathbb{C} ; \eta^{q}=-1\right\} \quad\left(\subseteq \mathbb{Q}_{2 q}\right) .
$$

Then $\left\langle\tau_{l}\right\rangle$ acts in the usual way on $E_{2 q}^{-}$. Let $\mathcal{Z}$ be the set of orbits under this action. Since $G_{p} / G_{p}^{2 q}=\langle\bar{g}\rangle$, there is a bijection

$$
X_{2 q}^{-} \rightarrow E_{2 q}^{-}: \chi \mapsto \chi(g)
$$

which induces the bijection

$$
\mathcal{Y} \rightarrow \mathcal{Z}: Y=\left\langle\tau_{l}\right\rangle \circ \chi_{1} \mapsto Z=\left\langle\tau_{l}\right\rangle\left(\chi_{1}(g)\right) .
$$

The system (6) defining the space $V_{Y, g}$ can be written as

$$
\sum_{j=0}^{q-1} \overline{T_{l}\left(\eta^{j-k}\right)} \bar{\Phi}_{j}=\overline{0}, \quad k=0, \ldots,|Z|-1,
$$

with $\eta \in Z$ arbitrary. By the systems (8) belonging to the orbits $Z$, the manifold $M_{l, g}$ is defined in an invariant way.

Lemma 2. Let $q$ be odd and suppose that the elements $g \in G_{p}$ are always chosen such that Assumption B of the Introduction holds. Then $M_{l, g}$ is independent of the choice of $g$ and $p$.

Proof. One argues as in the case of Lemma 1, but the role of $E_{2 q}^{-}$is played by $E_{q}=\left\{\eta \in \mathbb{C} ; \eta^{q}=1\right\}$; and in (8), $Z$ means an orbit of $\left\langle\tau_{l}\right\rangle$ on $E_{q}$.

If $Z$ is an orbit on $E_{2 q}^{-}$(on $E_{q}$, resp.), put

$$
V_{Z}=\left\{\bar{\Phi} \in \mathbb{F}_{l}^{q} ; \bar{\Phi} \text { satisfies (8) }\right\} \quad \text { and } \quad M_{l}=\bigcup\left(V_{Z} ; Z \in \mathcal{Z}\right) .
$$

In the situation of Lemmas 1 and 2 we have

$$
M_{l, g}=M_{l} \text {. }
$$

The spaces $V_{Z}$ defining the manifold $M_{l}$ have dimension $q-|Z|$, in accordance with Theorem 2 . We have shown: 
TheOREm 3. Let $q \in \mathbb{N}, l$ an odd prime, $l \nmid q, l \nmid \varphi(q)$. Suppose that for each prime number $p, p \equiv 2 q+1 \bmod 4 q, p>2 q+1$, the element $g$ is chosen such that Assumption A of the Introduction holds. Then there exists a linear manifold $M_{l} \subseteq \mathbb{F}_{l}^{q}$ with the following property: $C_{2 q} h_{2 q}^{-}(p) \equiv 0 \bmod l$ if and only if $\bar{\Phi}(g) \in M_{l}$.

This assertion remains valid if "Assumption A" is replaced by "Assumption B".

3. Special cases of systems of equations. The foregoing section sets the following task: bring the systems (8) describing $M_{l}$ into a form which is as explicit as possible. We shall do this in some special cases (e.g., for all $q \leq 6)$ and discuss the choice of these special cases.

(I) The case $\tau_{l}=\mathrm{id}$. Let $\tau_{l}=\mathrm{id}$, which means $l \equiv 1 \bmod 2 q$. Here $l$ splits completely in $\mathbb{Q}_{2 q}$ and $T_{l}=$ id. The set $\left\{\bar{\eta} \in \mathcal{O}_{2 q} / \mathfrak{L} ; \eta \in E_{2 q}^{-}\right\} \quad(\{\bar{\eta} \in$ $\left.\mathcal{O}_{2 q} / \mathfrak{L} ; \eta \in E_{q}\right\}$ in the case of Assumption B) can be identified with $\bar{E}_{2 q}^{-}=$ $\left\{w \in \mathbb{F}_{l} ; w^{q}=-1\right\}\left(\bar{E}_{q}=\left\{w \in \mathbb{F}_{l} ; w^{q}=1\right\}\right.$, resp.). The systems (8) take the form

$$
\sum_{j=0}^{q-1} w^{j} \bar{\Phi}_{j}=\overline{0} .
$$

We obtain: The prime $l$ divides $C_{2 q} h_{2 q}^{-}$if and only if equation (9) holds for at least one $w \in \bar{E}_{2 q}^{-}\left(\bar{E}_{q}\right.$, resp.). In the case of Assumption B this assertion was just the content of Theorem 4 in [4].

Suppose now that $\tau_{l} \neq$ id has a small order. Then $[L: \mathbb{Q}]$ is large and the elements $T_{l}\left(\eta^{j-k}\right) \in \mathcal{O}_{L}$ occurring in (8) are irrationalities of high degree, in general. It seems to be difficult to identify $\overline{T_{l}\left(\eta^{j-k}\right)} \in \mathcal{O}_{L} / \mathfrak{l}$ with an appropriate element of $\mathbb{F}_{l}=\mathbb{Z} / l \mathbb{Z}$ in this general context. For instance, let $l \equiv-1 \bmod 2 q$, which implies $\operatorname{ord}\left(\tau_{l}\right)=2$. If $\operatorname{ord}(\eta)=2 q$, the element $T_{l}(\eta)=\eta+\eta^{-1}$ generates the maximal real subfield of $\mathbb{Q}_{2 q}$. Apparently, the minimal polynomial $P$ of $\eta+\eta^{-1}$ over $\mathbb{Q}$ is not explicitly known (in general); the zeros of $\bar{P}$ in $\mathbb{F}_{l}$ are even less known. But these zeros occur, arranged in some way, as coefficients of equations (8). This discussion suggests to investigate the case when $\operatorname{ord}\left(\tau_{l}\right)$ is large, rather. Indeed, we shall only consider examples with $\operatorname{ord}\left(\tau_{l}\right) \in\{\varphi(2 q), \varphi(2 q) / 2\}$.

(II) The case $\operatorname{ord}\left(\tau_{l}\right)=\varphi(2 q)$. Here $\operatorname{Gal}\left(\mathbb{Q}_{2 q} / \mathbb{Q}\right)=\left\langle\tau_{l}\right\rangle$ is cyclic, which requires that $q \in\{1,2\}$ or that $q$ is an odd prime power. For $q=1$, $\Phi_{0}=C_{2 q} h_{2 q}^{-}$and (8) reads $\bar{\Phi}_{0}=\overline{0}$. If $q=2$, the set $E_{4}^{-}=\{ \pm \sqrt{-1}\}$ consists of a unique orbit, and (8) means $\bar{\Phi}_{0}=\bar{\Phi}_{1}=\overline{0} \in \mathbb{F}_{l}$. Therefore let $q=n^{r}$, $n \geq 3$ prime, $r \geq 1$. Furthermore, let Assumption $\mathrm{B}$ of the Introduction hold. Put $Z_{s}=\left\{\eta \in E_{q} ; \operatorname{ord}(\eta)=n^{s}\right\}, s=0,1, \ldots, r$. Then $\left|Z_{s}\right|=\varphi\left(n^{s}\right)$, 
and $\mathcal{Z}=\left\{Z_{0}, Z_{1}, \ldots, Z_{r}\right\}$. For an element $\eta \in E_{q}$,

$$
T_{l}(\eta)= \begin{cases}0 & \text { if } \eta \notin Z_{0} \cup Z_{1}, \\ -q / n & \text { if } \eta \in Z_{1}, \\ q-q / n & \text { if } \eta \in Z_{0} .\end{cases}
$$

The system (8) belonging to $Z_{0}$ is

$$
\bar{\Phi}_{0}+\bar{\Phi}_{1}+\ldots+\bar{\Phi}_{q-1}=\overline{0} .
$$

Let $s \geq 1$ and $\eta \in Z_{s}$ be arbitrary. Then the system (8) attached to $Z_{s}$ takes the form

$$
\begin{aligned}
\overline{n-1} \bar{\Phi}_{k}-\sum\left(\bar{\Phi}_{j} ; \eta^{j-k} \in Z_{1}, j \in\{0, \ldots, q-1\}\right) & =\overline{0}, \\
& k=0, \ldots, \varphi\left(n^{s}\right)-1 .
\end{aligned}
$$

Let us inspect the particular case $s=r \geq 1$. Here (10) reads

$\bar{n} \bar{\Phi}_{k}=\sum\left(\bar{\Phi}_{j} ; j \equiv k \bmod q / n, j \in\{0, \ldots, q-1\}\right), \quad k=0, \ldots, \varphi(q)-1 ;$

this system can be transformed into

$$
\bar{\Phi}_{j}=\bar{\Phi}_{k}, \quad k=0, \ldots, q / n-1, j=0, \ldots, q-1, j \equiv k \bmod q / n .
$$

If $r=1$ we obtain: Let $q$ be an odd prime, $l \nmid q, l \nmid q-1$. Then $l$ divides $C_{2 q} h_{2 q}^{-}$if and only if $\bar{\Phi}_{0}+\ldots+\bar{\Phi}_{q-1}=\overline{0}$ or $\bar{\Phi}_{0}=\bar{\Phi}_{1}=\ldots=\bar{\Phi}_{q-1}$. This statement is contained in Theorem 3 of [4].

In the remainder of this section $\operatorname{ord}\left(\tau_{l}\right)=\varphi(2 q) / 2$. Again, we restrict our interest to the simplest cases: viz., $q \geq 3$ prime, $q=2^{r}$, and $q=6$.

(III) The case $\operatorname{ord}\left(\tau_{l}\right)=(q-1) / 2, q \geq 3$ prime. Let Assumption B of the Introduction hold. We put

$$
Q=\{k \in \mathbb{Z} ; q \nmid k, k \text { a quadratic residue } \bmod q\}
$$

and

$$
N=\{k \in \mathbb{Z} ; q \nmid k, k \notin Q\} .
$$

Moreover, let $q^{*}=q$ if $q \equiv 1 \bmod 4$, and $q^{*}=-q$ if $q \equiv 3 \bmod 4$. Then $\left\langle\tau_{l}\right\rangle=\left\{\tau_{k} ; k \in Q\right\}$, and $L=\mathbb{Q}\left(\sqrt{q^{*}}\right)$. Take an element $\eta \in E_{q} \backslash\{1\}$. The set $E_{q}$ splits into the orbits

$$
Z_{1}=\{1\}, \quad Z_{2}=\left\{\eta^{k} ; k \in Q\right\}, \quad Z_{3}=\left\{\eta^{k} ; k \in N\right\} .
$$

By means of Gauss sums we obtain (cf. [1], p. 195)

$$
T_{l}\left(\eta^{k}\right)= \begin{cases}(q-1) / 2 & \text { if } q \mid k, \\ \left(-1+\sqrt{q^{*}}\right) / 2 & \text { if } k \in Q, \\ \left(-1-\sqrt{q^{*}}\right) / 2 & \text { if } k \in N .\end{cases}
$$


Here $\sqrt{q^{*}}$ depends on the choice of $\eta$. The elements $\overline{-1+\sqrt{q^{*}}}, \overline{-1-\sqrt{q^{*}}}$ of $\mathcal{O}_{L} / \mathfrak{l}$ can be identified with the zeros $w, w^{\prime}$ in $\mathbb{F}_{l}$ of the equation

$$
w^{2}+\overline{2} w+\overline{1-q^{*}}=\overline{0} .
$$

The system (8) belonging to $Z_{1}$ is $\bar{\Phi}_{0}+\ldots+\bar{\Phi}_{q-1}=\overline{0}$. For the orbit $Z_{2}$ it reads

$$
\overline{q-1} \bar{\Phi}_{k}+\sum_{\substack{j=0 \\ j-k \in Q}}^{q-1} w \bar{\Phi}_{j}+\sum_{\substack{j=0 \\ j-k \in N}}^{q-1} w^{\prime} \bar{\Phi}_{j}=\overline{0}, \quad k=0, \ldots,(q-3) / 2 .
$$

The corresponding system for $Z_{3}$ arises from (11) by interchanging $w$ and $w^{\prime}$.

(IV) The case $\operatorname{ord}\left(\tau_{l}\right)=q / 2, q=2^{r}$. Let the Assumption A of the Introduction hold. We may suppose that $q \geq 4$. In general, only two groups $\left\langle\tau_{l}\right\rangle$ can occur, viz., $\left\langle\tau_{l}\right\rangle=\left\langle\tau_{5}\right\rangle$, if $l \equiv 5 \bmod 8$, and $\left\langle\tau_{l}\right\rangle=\left\langle\tau_{-5}\right\rangle$, if $l \equiv$ $3 \bmod 8$. In the case $q=4$ there is an additional group, viz., $\left\langle\tau_{7}\right\rangle=\left\langle\tau_{-1}\right\rangle$.

We consider the case $\left\langle\tau_{l}\right\rangle=\left\langle\tau_{5}\right\rangle$ first. The set $E_{2 q}^{-}$consists of two orbits $Z_{1}, Z_{2}$ of length $\left|Z_{1}\right|=\left|Z_{2}\right|=q / 2$. Furthermore, $L=\mathbb{Q}(\sqrt{-1})$, and for $\eta \in E_{2 q}^{-}, k \in \mathbb{Z}$

$$
T_{l}\left(\eta^{k}\right)= \begin{cases}(q / 2) \eta^{k} & \text { if } k \equiv 0 \bmod q / 2, \\ 0 & \text { otherwise. }\end{cases}
$$

We identify $\overline{\eta^{q / 2}}=\overline{\sqrt{-1}} \in \mathcal{O}_{L} / \mathfrak{l}$ with the corresponding root $w \in \mathbb{F}_{l}$ of the equation $w^{2}+\overline{1}=\overline{0}$. Then the equations (8) for $Z_{1}$ take the form

$$
\bar{\Phi}_{k+q / 2}=w \bar{\Phi}_{k}, \quad k=0, \ldots, q / 2-1 .
$$

In the equations for $Z_{2}, w$ must be replaced by $-w$.

If $\left\langle\tau_{l}\right\rangle=\left\langle\tau_{-5}\right\rangle$, there are also two orbits $Z_{1}, Z_{2}$ of equal length. Here $L=\mathbb{Q}(\sqrt{-2})$, and for $\eta \in E_{2 q}^{-}, k \in \mathbb{Z}$,

$$
T_{l}\left(\eta^{k}\right)= \begin{cases}(q / 4)\left(\eta^{k}+\eta^{3 k}\right) & \text { if } k \equiv 0 \bmod q / 4, \\ 0 & \text { otherwise. }\end{cases}
$$

Let $w \in \mathbb{F}_{l}$ be a root of $w^{2}+\overline{2}=\overline{0}$. Then the first system (8) reads

$$
\begin{aligned}
\bar{\Phi}_{k+q / 2} & =-\bar{\Phi}_{k}+w \bar{\Phi}_{k+q / 4}, \quad k=0, \ldots, q / 4-1 . \\
\bar{\Phi}_{k+3 q / 4} & =w \bar{\Phi}_{k}-\bar{\Phi}_{k+q / 4},
\end{aligned}
$$

In the second system (8) the root $w$ is replaced by $-w$.

Finally, if $q=4$ and $l \equiv 7 \bmod 8$, there are also two orbits of equal length, and $L=\mathbb{Q}(\sqrt{2})$. Let $w \in \mathbb{F}_{l}$ be a root of $w^{2}-\overline{2}=\overline{0}$. The first system (8) is

$$
\left\{\begin{array}{l}
\bar{\Phi}_{2}=w \bar{\Phi}_{1}-\bar{\Phi}_{0} \\
\bar{\Phi}_{3}=\bar{\Phi}_{1}-w \bar{\Phi}_{0}
\end{array}\right.
$$

Again, the substitution $w \mapsto-w$ yields the second system. 
(V) The case $q=6$. If $\tau_{l} \neq \mathrm{id}$, the order of $\tau_{l}$ is 2 , and the cases $l \equiv 5,7,11 \bmod 12$ must be distinguished. All of them are treated similarly, hence we pick out the case $l \equiv 5 \bmod 12$ only. Let $\eta \in E_{12}^{-}, \operatorname{ord}(\eta)=12$. There are four orbits: $Z_{1}=\left\{\eta^{3}\right\}, Z_{2}=\left\{\eta^{9}\right\}, Z_{3}=\left\{\eta, \eta^{5}\right\}, Z_{4}=\left\{\eta^{7}, \eta^{11}\right\}$. Moreover, $\eta^{3}=\sqrt{-1}$ and $L=\mathbb{Q}(\sqrt{-1})$. By means of the relation $\eta^{4}=\eta^{2}-1$ arising from the 12 th cyclotomic polynomial, one obtains

$$
T_{l}\left(\eta^{k}\right)= \begin{cases}\eta^{3 k} & \text { if }(k, 12)=1, \\ 2 \eta^{k} & \text { if } k \equiv \pm 3 \bmod 12 \\ -1 & \text { if } k \equiv \pm 4 \bmod 12 \\ 1 & \text { if } k \equiv \pm 2 \bmod 12 .\end{cases}
$$

Let $w$ be a root of $w^{2}+\overline{1}=\overline{0}$. The system (8) of $Z_{1}$ consists of the equation

$$
\bar{\Phi}_{0}-\bar{\Phi}_{2}+\bar{\Phi}_{4}=w\left(\bar{\Phi}_{1}-\bar{\Phi}_{3}+\bar{\Phi}_{5}\right) .
$$

In the case of $Z_{3}$ there are two equations:

$$
\begin{aligned}
& \overline{2 \Phi}_{0}+\bar{\Phi}_{2}-\bar{\Phi}_{4}=w\left(\bar{\Phi}_{1}+\overline{2 \Phi}_{3}+\bar{\Phi}_{5}\right), \\
& \bar{\Phi}_{0}-\bar{\Phi}_{2}-\overline{2 \Phi}_{4}=w\left(\overline{2 \Phi}_{1}+\bar{\Phi}_{3}-\bar{\Phi}_{5}\right) .
\end{aligned}
$$

The substitution $w \mapsto-w$ yields the systems (8) belonging to $Z_{2}$ and $Z_{4}$.

R e mark. From the systems of equations occurring in cases (III)-(V) one can deduce quadratic congruences $\bmod l$ which are very convenient in practice. For instance, the equations (12) imply

$$
2 \Phi_{0}^{2} \equiv\left(\Phi_{1}-\Phi_{3}\right)^{2} \bmod l, \quad 2 \Phi_{1}^{2} \equiv\left(\Phi_{0}+\Phi_{2}\right)^{2} \bmod l .
$$

4. Numerical results. Let the above notations hold. We are interested in applying Theorem 3 to $q=1,2, \ldots, 6$. The hypothesis $l \nmid \varphi(q)$ of this theorem is meaningless here, since $\varphi(q)$ is a power of 2 . In the sequel we must exclude the case that $l$ divides $C_{2 q}$. For this reason we collect up the pairs $\left(q, f_{2 q}\right), q \leq 6$, for which a prime $l \geq 3$ divides $C_{2 q}$ (cf. formula (3)).

$$
\begin{aligned}
& l=3: \quad\left(q, f_{2 q}\right) \in\{(1,2),(3,2),(3,6),(4,2),(5,2),(5,10)\} ; \\
& l=5: \quad\left(q, f_{2 q}\right) \in\{(2,4),(4,4),(6,4),(6,12)\} ; \\
& l=7: \quad\left(q, f_{2 q}\right)=(3,3) ; \\
& l=11:\left(q, f_{2 q}\right)=(5,10) ; \\
& l=13:\left(q, f_{2 q}\right)=(6,12) ; \\
& l=31:\left(q, f_{2 q}\right)=(5,5) .
\end{aligned}
$$

In what follows let Assumption A hold for even $q$ 's and Assumption B for odd ones. The set $\mathcal{Z}$ consists of all orbits of $\left\langle\tau_{l}\right\rangle$ on $E_{2 q}^{-}$(on $E_{q}$, resp.) and, as above,

$$
M_{l}=\bigcup\left(V_{Z} ; Z \in \mathcal{Z}\right) .
$$


Let $p$ denote a prime, $p \equiv 2 q+1 \bmod 4 q, p>2 q+1$. If $l$ divides $C_{2 q}=$ $C_{2 q}(p)$, the vector $\bar{\Phi}=\bar{\Phi}(g)$ is in $M_{l}$, of course. However, if $p$ runs through all primes with $l \nmid C_{2 q}$, it could happen that the excess vectors $\bar{\Phi}$ were equally distributed in the space $\mathbb{F}_{l}^{q}$. Suppose this is true. Then the number

$$
m_{l}=\left|M_{l}\right| /\left|\mathbb{F}_{l}^{q}\right|=\left|M_{l}\right| / l^{q}
$$

is the probability that $l$ divides the class number $h_{2 q}^{-}(p)$, by Theorem 3 .

In order to compute $m_{l}$ one has to determine the cardinality of $M_{l}$. This can be done by means of the well-known sieve formula (cf. [1], p. 123)

$$
\begin{aligned}
M_{l}= & \sum\left(\left|V_{Z}\right| ; Z \in \mathcal{Z}\right)-\sum\left(\left|V_{Z} \cap V_{Z^{\prime}}\right| ;\left\{Z, Z^{\prime}\right\} \subseteq \mathcal{Z}\right) \\
& +\sum\left(\left|V_{Z} \cap V_{Z^{\prime}} \cap V_{Z^{\prime \prime}}\right| ;\left\{Z, Z^{\prime}, Z^{\prime \prime}\right\} \subseteq \mathcal{Z}\right)-\ldots
\end{aligned}
$$

According to Theorem $2,\left|V_{Z}\right|=l^{q-|Z|}$ for all $Z \in \mathcal{Z}$. From the proof of Theorem 2 it is clear that

$$
\bigcap\left(V_{Z} ; Z \in \mathcal{Z}\right)=\{0\}
$$

i.e., the union of all systems (8) forms a linearly independent system of equations. For these reasons (13) yields

$$
\begin{aligned}
\left|M_{l}\right|= & \sum\left(l^{q-|Z|} ; Z \in \mathcal{Z}\right)-\sum\left(l^{q-|Z|-\left|Z^{\prime}\right|} ;\left\{Z, Z^{\prime}\right\} \subseteq \mathcal{Z}\right) \\
& +\sum\left(l^{q-|Z|-\left|Z^{\prime}\right|-\left|Z^{\prime \prime}\right|} ;\left\{Z, Z^{\prime}, Z^{\prime \prime}\right\} \subseteq \mathcal{Z}\right)-\ldots
\end{aligned}
$$

Moreover, if all orbits $Z \in \mathcal{Z}$ have the same length $|Z|=z$, (15) takes the simplified form

$$
\left|M_{l}\right|=l^{q}\left(1-\left(1-1 / l^{z}\right)^{q / z}\right) .
$$

If $q$ is an odd prime number, one orbit has length 1 and the remaining ones the same length $z$. From (16) we deduce for this situation

$$
\left|M_{l}\right|=l^{q-1}\left(1+(l-1)\left(1-1 / l^{z}\right)^{(q-1) / z}\right) .
$$

The values of $m_{l}$ given in Table 1 have been found by means of (15)-(17).

We put

$$
P=\{p ; p \text { prime }, p<500000, p \equiv 2 q+1 \bmod 4 q, p>2 q+1\}
$$

and

$$
n_{l}=\left|\left\{p \in P ; l \mid h_{2 q}^{-}(p)\right\}\right| /|P| .
$$

For small primes $l \geq 3, l \nmid q, l \nmid C_{2 q}, q \leq 6$, the number $n_{l}$ can serve as an approximation of the probability that $l$ divides $h_{2 q}^{-}(p)$. In the few cases where $l$ divides a number $C_{2 q}=C_{2 q}(p)$ (cf. the above list), we define $n_{l}$ as

$$
n_{l}=\left|\left\{p \in P ; l \nmid C_{2 q}(p), l \mid h_{2 q}^{-}(p)\right\}\right| /\left|\left\{p \in P ; l \nmid C_{2 q}(p)\right\}\right| .
$$


Table 1

l-divisibility of $h_{2}^{-}(p)$ for $p<500000$; total number of $p$ 's: 20805

\begin{tabular}{cccrcc}
\hline$l$ & $n_{l}$ & $m_{l}$ & $l$ & $n_{l}$ & $m_{l}$ \\
\hline $3^{*}$ & 0.4063 & 0.3333 & 5 & 0.2313 & 0.2000 \\
7 & 0.1634 & 0.1429 & 11 & 0.0992 & 0.0909 \\
13 & 0.0817 & 0.0769 & 17 & 0.0636 & 0.0588 \\
19 & 0.0545 & 0.0526 & 23 & 0.0453 & 0.0435 \\
29 & 0.0343 & 0.0345 & 31 & 0.0344 & 0.0323 \\
37 & 0.0263 & 0.0270 & 41 & 0.0256 & 0.0244 \\
43 & 0.0246 & 0.0233 & 47 & 0.0219 & 0.0213 \\
53 & 0.0192 & 0.0189 & 59 & 0.0175 & 0.0169 \\
61 & 0.0170 & 0.0164 & 67 & 0.0146 & 0.0149 \\
71 & 0.0158 & 0.0141 & 73 & 0.0129 & 0.0137 \\
79 & 0.0146 & 0.0127 & 83 & 0.0125 & 0.0120 \\
89 & 0.0115 & 0.0112 & 97 & 0.0106 & 0.0103 \\
\hline
\end{tabular}

l-divisibility of $h_{4}^{-}(p)$ for $p<500000$; total number of $p$ 's: 10396

\begin{tabular}{rccrcc}
\hline$l$ & $n_{l}$ & $m_{l}$ & $l$ & $n_{l}$ & $m_{l}$ \\
\hline 3 & 0.1293 & 0.1111 & 7 & 0.0189 & 0.0204 \\
11 & 0.0082 & 0.0083 & 13 & 0.1513 & 0.1479 \\
17 & 0.1238 & 0.1142 & 19 & 0.0036 & 0.0028 \\
23 & 0.0009 & 0.0019 & 29 & 0.0676 & 0.0678 \\
31 & 0.0013 & 0.0010 & 37 & 0.0526 & 0.0533 \\
41 & 0.0518 & 0.0482 & 43 & 0.0007 & 0.0005 \\
47 & 0.0001 & 0.0005 & 53 & 0.0374 & 0.0374 \\
59 & 0.0003 & 0.0003 & 61 & 0.0368 & 0.0325 \\
67 & 0.0002 & 0.0002 & 71 & 0.0002 & 0.0002 \\
73 & 0.0261 & 0.0272 & 79 & 0.0003 & 0.0002 \\
83 & 0.0003 & 0.0001 & 89 & 0.0209 & 0.0223 \\
97 & 0.0187 & 0.0205 & & & \\
\hline
\end{tabular}

In Table 1 we display both "probabilities" $n_{l}$ and $m_{l}$ for $q \leq 6$ and $3 \leq$ $l<100, l \nmid q$. The primes $l$ for which $l \mid C_{2 q}(p)$ can occur are distinguished by an asterisk.

If $q$ is odd, the number $C_{2 q} h_{2 q}^{-}$is divisible by $C_{2} h_{2}^{-}$. Theorem 3 and formula (2) yield the following

Corollary. Let $q \geq 1$ be odd, $p$ prime, $p \equiv 2 q+1 \bmod 4 q, p>2 q+1$. Let $l \geq 3$ be a prime, $l \nmid q, l \nmid q-1$. Then $l$ divides $C_{2 q} h_{2 q}^{-} /\left(C_{2} h_{2}^{-}\right)$if and only if the vector $\bar{\Phi} \in \mathbb{F}_{l}^{q}$ is in the linear manifold

$$
M_{l}^{*}=\bigcup\left(V_{Z} ; Z \in \mathcal{Z}, Z \neq\{1\}\right) .
$$


Table 1 (cont.)

l-divisibility of $h_{6}^{-}(p)$ for $p<500000$; total number of $p$ 's: 10402

\begin{tabular}{rccccccccc}
\hline$l$ & $n_{l}$ & $m_{l}$ & $n_{l}^{*}$ & $m_{l}^{*}$ & $l$ & $n_{l}$ & $m_{l}$ & $n_{l}^{*}$ & $m_{l}^{*}$ \\
\hline 5 & 0.2701 & 0.2320 & 0.0386 & 0.0400 & $7^{*}$ & 0.4104 & 0.3703 & 0.2899 & 0.2653 \\
11 & 0.1055 & 0.0984 & 0.0074 & 0.0083 & 13 & 0.2269 & 0.2135 & 0.1578 & 0.1479 \\
17 & 0.0663 & 0.0621 & 0.0030 & 0.0035 & 19 & 0.1543 & 0.1497 & 0.1073 & 0.1025 \\
23 & 0.0480 & 0.0453 & 0.0010 & 0.0019 & 29 & 0.0349 & 0.0356 & 0.0012 & 0.0012 \\
31 & 0.0976 & 0.0937 & 0.0673 & 0.0635 & 37 & 0.0822 & 0.0789 & 0.0567 & 0.0533 \\
41 & 0.0254 & 0.0250 & 0.0004 & 0.0006 & 43 & 0.0705 & 0.0682 & 0.0473 & 0.0460 \\
47 & 0.0221 & 0.0217 & 0.0004 & 0.0005 & 53 & 0.0184 & 0.0192 & 0.0003 & 0.0004 \\
59 & 0.0195 & 0.0172 & 0.0002 & 0.0003 & 61 & 0.0477 & 0.0484 & 0.0316 & 0.0325 \\
67 & 0.0441 & 0.0441 & 0.0289 & 0.0296 & 71 & 0.0174 & 0.0143 & 0.0002 & 0.0002 \\
73 & 0.0385 & 0.0405 & 0.0261 & 0.0272 & 79 & 0.0392 & 0.0375 & 0.0248 & 0.0252 \\
83 & 0.0138 & 0.0122 & 0.0002 & 0.0001 & 89 & 0.0130 & 0.0114 & 0.0001 & 0.0001 \\
97 & 0.0327 & 0.0306 & 0.0225 & 0.0205 & & & & & \\
\hline
\end{tabular}

$l$-divisibility of $h_{8}^{-}(p)$ for $p<500000$; total number of $p$ 's: 5165

\begin{tabular}{cccccc}
\hline$l$ & $n_{l}$ & $m_{l}$ & $l$ & $n_{l}$ & $m_{l}$ \\
\hline $3^{*}$ & 0.2151 & 0.2099 & $5^{*}$ & 0.0794 & 0.0784 \\
7 & 0.0414 & 0.0404 & 11 & 0.0170 & 0.0165 \\
13 & 0.0112 & 0.0118 & 17 & 0.2290 & 0.2153 \\
19 & 0.0048 & 0.0055 & 23 & 0.0043 & 0.0038 \\
29 & 0.0031 & 0.0024 & 31 & 0.0017 & 0.0021 \\
37 & 0.0019 & 0.0015 & 41 & 0.0931 & 0.0940 \\
43 & 0.0019 & 0.0011 & 47 & 0.0010 & 0.0009 \\
53 & 0.0004 & 0.0007 & 59 & 0.0002 & 0.0006 \\
61 & 0.0002 & 0.0005 & 67 & 0.0010 & 0.0004 \\
71 & 0.0002 & 0.0004 & 73 & 0.0511 & 0.0537 \\
79 & 0.0004 & 0.0003 & 83 & 0.0008 & 0.0003 \\
89 & 0.0409 & 0.0442 & 97 & 0.0451 & 0.0406 \\
\hline
\end{tabular}

In view of the Corollary we also render the numbers

$$
n_{l}^{*}=\left|\left\{p \in P ; l \mid\left(h_{2 q}^{-}(p) / h_{2}^{-}(p)\right)\right\}\right| /|P|
$$

and

$$
m_{l}^{*}=\left|M_{l}^{*}\right| / l^{q}
$$

in Table 1 , for $q=3,5$. If $l$ divides some quotient $C_{2 q} / C_{2}$, the definition of $n_{l}^{*}$ has been modified appropriately.

Let $q=6$. Then $C_{4} h_{4}^{-}$divides $C_{12} h_{12}^{-}$. Again, $l$ divides the quotient $C_{12} h_{12}^{-} /\left(C_{4} h_{4}^{-}\right)$if and only if $\bar{\Phi}$ is in a certain linear manifold $M_{l}^{*} \subseteq \mathbb{F}_{l}^{6}$. Table 1 contains $m_{l}^{*}=\left|M_{l}^{*}\right| / l^{6}$ and the comparative figure $n_{l}^{*}$. 
Table 1 (cont.)

$l$-divisibility of $h_{10}^{-}(p)$ for $p<500000$; total number of $p$ 's: 5208

\begin{tabular}{cccccccccc}
\hline$l$ & $n_{l}$ & $m_{l}$ & $n_{l}^{*}$ & $m_{l}^{*}$ & $l$ & $n_{l}$ & $m_{l}$ & $n_{l}^{*}$ & $m_{l}^{*}$ \\
\hline $3^{*}$ & 0.4181 & 0.3416 & 0.0054 & 0.0123 & 7 & 0.1674 & 0.1432 & 0.0006 & 0.0004 \\
$11^{*}$ & 0.4203 & 0.3791 & 0.3514 & 0.3170 & 13 & 0.0816 & 0.0770 & 0.0000 & 0.0000 \\
17 & 0.0588 & 0.0588 & 0.0000 & 0.0000 & 19 & 0.0613 & 0.0579 & 0.0056 & 0.0055 \\
23 & 0.0432 & 0.0435 & 0.0000 & 0.0000 & 29 & 0.0313 & 0.0368 & 0.0021 & 0.0024 \\
$31^{*}$ & 0.1602 & 0.1512 & 0.1281 & 0.1229 & 37 & 0.0244 & 0.0270 & 0.0000 & 0.0000 \\
41 & 0.1171 & 0.1161 & 0.0916 & 0.0940 & 43 & 0.0246 & 0.0233 & 0.0000 & 0.0000 \\
47 & 0.0236 & 0.0213 & 0.0000 & 0.0000 & 53 & 0.0173 & 0.0189 & 0.0000 & 0.0000 \\
59 & 0.0207 & 0.0175 & 0.0010 & 0.0006 & 61 & 0.0762 & 0.0793 & 0.0618 & 0.0640 \\
67 & 0.0134 & 0.0149 & 0.0000 & 0.0000 & 71 & 0.0672 & 0.0685 & 0.0545 & 0.0552 \\
73 & 0.0113 & 0.0137 & 0.0000 & 0.0000 & 79 & 0.0180 & 0.0130 & 0.0004 & 0.0003 \\
83 & 0.0132 & 0.0120 & 0.0000 & 0.0000 & 89 & 0.0119 & 0.0115 & 0.0006 & 0.0003 \\
97 & 0.0117 & 0.0103 & 0.0000 & 0.0000 & & & & & \\
\hline
\end{tabular}

$l$-divisibility of $h_{12}^{-}(p)$ for $p<500000$; total number of $p$ 's: 5191

\begin{tabular}{cccccccccc}
\hline$l$ & $n_{l}$ & $m_{l}$ & $n_{l}^{*}$ & $m_{l}^{*}$ & $l$ & $n_{l}$ & $m_{l}$ & $n_{l}^{*}$ & $m_{l}^{*}$ \\
\hline $5^{*}$ & & & 0.0820 & 0.0784 & 7 & 0.0543 & 0.0600 & 0.0358 & 0.0404 \\
11 & 0.0235 & 0.0246 & 0.0171 & 0.0165 & $13^{*}$ & 0.4038 & 0.3814 & 0.2993 & 0.2740 \\
17 & 0.1310 & 0.1203 & 0.0060 & 0.0069 & 19 & 0.0100 & 0.0083 & 0.0062 & 0.0055 \\
23 & 0.0056 & 0.0057 & 0.0046 & 0.0038 & 29 & 0.0657 & 0.0700 & 0.0025 & 0.0024 \\
31 & 0.0029 & 0.0031 & 0.0017 & 0.0021 & 37 & 0.1516 & 0.1516 & 0.1063 & 0.1038 \\
41 & 0.0541 & 0.0493 & 0.0012 & 0.0012 & 43 & 0.0025 & 0.0016 & 0.0019 & 0.0011 \\
47 & 0.0008 & 0.0014 & 0.0006 & 0.0009 & 53 & 0.0364 & 0.0381 & 0.0006 & 0.0007 \\
59 & 0.0006 & 0.0009 & 0.0004 & 0.0006 & 61 & 0.0896 & 0.0944 & 0.0584 & 0.0640 \\
67 & 0.0000 & 0.0007 & 0.0000 & 0.0004 & 71 & 0.0006 & 0.0006 & 0.0002 & 0.0004 \\
73 & 0.0746 & 0.0794 & 0.0516 & 0.0537 & 79 & 0.0008 & 0.0005 & 0.0002 & 0.0003 \\
83 & 0.0006 & 0.0004 & 0.0002 & 0.0003 & 89 & 0.0223 & 0.0226 & 0.0000 & 0.0003 \\
97 & 0.0599 & 0.0603 & 0.0403 & 0.0406 & & & & & \\
\hline
\end{tabular}

In Table 2 we have collected up the relative class numbers $h_{12}^{-}(p)$ for all $p<10000$ ( $p \equiv 13 \bmod 24$, of course).

Table 2. Relative class numbers $h_{12}^{-}$

\begin{tabular}{rrcrrr}
\hline$p$ & $h_{12}^{-}$ & $p$ & $h_{12}^{-}$ & $p$ & $h_{12}^{-}$ \\
\hline 13 & 1 & 37 & 1 & 61 & 1 \\
109 & 17 & 157 & 65 & 181 & 925 \\
229 & 221 & 277 & 272 & 349 & 1040 \\
373 & 305 & 397 & 832 & 421 & 925 \\
541 & 2257 & 613 & 2425 & 661 & 1053
\end{tabular}


Table 2 (cont.)

\begin{tabular}{|c|c|c|c|c|c|}
\hline$p$ & $h_{12}^{-}$ & $p$ & $h_{12}^{-}$ & $p$ & $h_{12}^{-}$ \\
\hline 709 & 12688 & 733 & 3645 & 757 & 157625 \\
\hline 829 & 26245 & 853 & 2516 & 877 & 22681 \\
\hline 997 & 1825 & 1021 & 3977 & 1069 & 13949 \\
\hline 1093 & 555185 & 1117 & 577405 & 1213 & 94357 \\
\hline 1237 & 42125 & 1381 & 166617 & 1429 & 288353 \\
\hline 1453 & 270725 & 1549 & 17725 & 1597 & 682541 \\
\hline 1621 & 1441557 & 1669 & 1512745 & 1693 & 314237 \\
\hline 1741 & 116285 & 1789 & 57616 & 1861 & 132977 \\
\hline 1933 & 24737 & 2029 & 3922321 & 2053 & 92537 \\
\hline 2221 & 1797497 & 2269 & 67625 & 2293 & 171593 \\
\hline 2341 & 1173037 & 2389 & 23725 & 2437 & 660857 \\
\hline 2557 & 514345 & 2677 & 1338949 & 2749 & 1112905 \\
\hline 2797 & 1502800 & 2917 & 300913 & 3037 & 469456 \\
\hline 3061 & 102245 & 3109 & 350649 & 3181 & 7938905 \\
\hline 3229 & 3985097 & 3253 & 9983713 & 3301 & 369313 \\
\hline 3373 & 7747909 & 3469 & 821881 & 3517 & 186004 \\
\hline 3541 & 152165 & 3613 & 2595125 & 3637 & 3896505 \\
\hline 3709 & 6131905 & 3733 & 20787845 & 3853 & 14944265 \\
\hline 3877 & 3801037 & 4021 & 849433 & 4093 & 37654825 \\
\hline 4261 & 570704 & 4357 & 1633360 & 4549 & 457145 \\
\hline 4597 & 1505969 & 4621 & 5254945 & 4789 & 3930768 \\
\hline 4813 & 3288745 & 4861 & 21461193 & 4909 & 5479825 \\
\hline 4933 & 24722117 & 4957 & 15291185 & 5077 & 601625 \\
\hline 5101 & 5343205 & 5197 & 623376 & 5413 & 2707549 \\
\hline 5437 & 1916217 & 5557 & 6719089 & 5581 & 1208453 \\
\hline 5653 & 8808669 & 5701 & 7036165 & 5749 & 6233305 \\
\hline 5821 & 907985 & 5869 & 1652813 & 6037 & 1839188 \\
\hline 6133 & 1254509 & 6229 & 5476409 & 6277 & 6378125 \\
\hline 6301 & 74076509 & 6373 & 7973593 & 6397 & 11072477 \\
\hline 6421 & 20553277 & 6469 & 8725853 & 6637 & 9356180 \\
\hline 6661 & 13352065 & 6709 & 1458500 & 6733 & 3908125 \\
\hline 6781 & 18425549 & 6829 & 12125605 & 6949 & 5479825 \\
\hline 6997 & 5553841 & 7069 & 43433797 & 7213 & 1275625 \\
\hline 7237 & 14537637 & 7309 & 5188433 & 7333 & 6472325 \\
\hline 7477 & 8024605 & 7549 & 2665345 & 7573 & 124889341 \\
\hline 7621 & 26335985 & 7669 & 345404785 & 7717 & 95208637 \\
\hline 7741 & 2900269 & 7789 & 10178869 & 7933 & 19589465 \\
\hline 8053 & 88674769 & 8101 & 20686509 & 8221 & 6688625 \\
\hline 8269 & 283411453 & 8293 & 14654925 & 8317 & 7268249 \\
\hline 8389 & 7384609 & 8461 & 5808245 & 8581 & 2116585 \\
\hline 8629 & 77909364 & 8677 & 550198737 & 8821 & 120093581 \\
\hline 8893 & 2169593 & 8941 & 43577965 & 9013 & 27373801 \\
\hline
\end{tabular}


Table 2 (cont.)

\begin{tabular}{rrrrrr}
\hline$p$ & $h_{12}^{-}$ & $p$ & $h_{12}^{-}$ & $p$ & $h_{12}^{-}$ \\
\hline 9109 & 1759504 & 9133 & 10980625 & 9157 & 2655065 \\
9181 & 4484077 & 9277 & 156931101 & 9349 & 20541845 \\
9397 & 22924681 & 9421 & 397973056 & 9613 & 406792061 \\
9661 & 44395585 & 9733 & 26450125 & 9781 & 34076653 \\
9829 & 7163125 & 9901 & 661365493 & 9949 & 15834377 \\
9973 & 286173589 & & & & \\
\hline
\end{tabular}

\section{References}

[1] T. M. Apostol, Introduction to Analytic Number Theory, Springer, New York 1976.

[2] S. I. Borevič und I. R. Šafarevič, Zahlentheorie, Birkhäuser, Basel 1966.

[3] K. Girstmair, The relative class numbers of imaginary cyclic fields of degrees 4, 6, 8, and 10, Math. Comp., to appear.

[4] - On the cosets of the 2q-power group in the unit group modulo $p$, Abh. Math. Sem. Univ. Hamburg 62 (1992), 217-232.

[5] H. Hasse, Über die Klassenzahl abelscher Zahlkörper (Nachdruck der ersten Auflage), Springer, Berlin 1985.

[6] R. H. Hudson, Class numbers of imaginary cyclic quartic fields and related quaternary systems, Pacific J. Math. 115 (1984), 129-142.

INSTITUT FÜR MATHEMATIK

UNIVERSITÄT INNSBRUCK

TECHNIKERSTRASSE $25 / 7$

A-6020 INNSBRUCK, ÖSTERREICH 